\title{
The Production of Terrestrial Meteorites - Moon Accretion and Lithopanspermia
}

\author{
Martin Beech ${ }^{1,2}$, Mark Comte ${ }^{2}$, Ian Coulson ${ }^{3}$ \\ ${ }^{1}$ Campion College, Department of Astronomy, The University of Regina, Regina, Canada \\ ${ }^{2}$ Department of Physics, The University of Regina, Regina, Canada \\ ${ }^{3}$ Department of Geology, The University of Regina, Regina, Canada \\ Email address: \\ beechm@uregina.ca (M. Beech),comte20m@uregina.ca (M. Comte), ian.coulson@uregina.ca (I. Coulson)

\section{To cite this article:} \\ Martin Beech, Mark Comte, Ian Coulson. The Production of Terrestrial Meteorites - Moon Accretion and Lithopanspermia. American \\ Journal of Astronomy and Astrophysics. Vol. 7, No. 1, 2019, pp. 1-9. doi: 10.11648/j.ajaa.20190701.11
}

Received: May 9, 2019; Accepted: June 12, 2019; Published: July 1, 2019

\begin{abstract}
The conditions under which terrestrial, impact-derived ejecta can be launched into cis-lunar space is studied. A numerical code is developed in order to follow the ablation and deceleration conditions of material ejected from the Earth's surface and outwards through the atmosphere. The deceleration filtering-effect imposed by Earth's atmosphere results in multimeter-sized, 5 to 20 meters across, fragments escaping into cis-lunar space being favored. Smaller fragments tend to be more rapidly decelerated than larger ones and are re-accreted by the Earth. The conditions under which Earth-ejected material might impact upon the Moon is additionally considered. It is found that for encounter speeds smaller than some $7 \mathrm{~km} / \mathrm{s}$, terrestrial meteorites might be expected to survive upon impact (that is they will not undergo shock melting) when encountering the Moon's regolith. It is argued that terrestrial meteorites may well survive, with identifiable features (fusion crust and mineralogy), for long periods of time within the lunar regolith (a result recently vindicated through the discovery of terrestrial material - launched during the late heavy bombardment - contained within a lunar impact breccia \#14321, collected during the Apollo 14 Moon landing mission). Further to this, the important role that terrestrial meteorites must have played in transporting microbial life to other potentially habitable locations within the solar system is discussed.
\end{abstract}

Keywords: Terrestrial Meteorites, Impact Craters, Impact Ejecta, Lithopanspermia

\section{Introduction}

The production of craters through asteroid and cometary nuclei impacts is a ubiquitous process that has operated within the solar system since its very birth, 4.56 billion years ago $[1,2]$. The Earth being no exception to this surfacesculpting process currently sports nearly 200 known impact craters $[2,3]$, and it must, since the time that it formed, have suffered many thousands of impacts. Geological processes, chiefly plate tectonics, having effectively removed evidence of many of these. Great quantities of energy are exchanged in the crater-producing process, with the kinetic energy of the impacting body being diverted into the compression, melting, vaporization, shock alteration and explosive dispersal of terrestrial material [1]. That impacts upon the Earth's surface can produce extensive ejecta blankets and, in some cases, extensive tektite strewn fields, has long been known [4], but what has received very little attention in the past is the fact that some of the ejecta produced during a cratering event can be released with speeds that exceed Earth's escape velocity. Such material can accordingly find its way into the inner solar system and thereby be accreted by the Moon and other planetary bodies. Indeed, with respect to the propagation of microbes into both interplanetary and interstellar space via lithopanspermia, the Earth is a critical object since it is known that microbial life has been abundant upon its surface for many billions of years. Upon this basis, therefore, it is not unreasonable to assume that terrestrial meteorites are likely to contain a temporary living cargo and be an important lithopanspermia vector. The Moon is the first potential port of call for all terrestrial impact ejecta capable of escaping from Earth's gravitational potential well, and thus the 
prospects for finding terrestrial material on the lunar surface are both high and potentially realizable in the near-term future $[5,6]$. This latter prospect being predicated upon the current plans of various national space agencies to return both autonomous rovers and astronauts to the lunar surface. Additionally, as inspiration for a new era of investigation, a recent analysis by Bellucci et al. [7] has found evidence for ancient terrestrial material being incorporated into a lunar breccia sample that was collected during the Apollo 14 mission in 1971.

That material can be exchanged between planetary bodies and the Moon is well documented with respect to the collection of both Lunar and Martian meteorites upon Earth's surface [8]. Indeed, of the 60,556 named and classified meteorites that have been collected worldwide, $227(0.4 \%)$ are Martian in origin and $360(0.6 \%)$ are from the Moon (data from the Meteoritical Bulletin Database, April, 2019) these numbers, of course, do not represent the true percentage distribution of meteorite types and/or transfer efficiencies; rather they are just a representation of such meteorites as have been found. There has been some speculation that perhaps several meteorites from Mercury could reside within the known meteorite collections, but to date none (except, perhaps for NWA 7325) have been unambiguously identified. Hermean meteorites could presumably masquerade as members of either the aubrite meteorite group or belong to one of the unspecified achondrite groups, with the key difficulty of recognition being that of identifying a specifically unique Hermean signature [9]. The collection of Martian and Lunar meteorites indicates that basaltic and ultramafic cumulate lithologies (in the case of Mars and potentially Mercury) and brecciacompacted regolith (in the case of the Moon) can be launched without complete fragmentation and/or vaporization during crater forming events. And, furthermore, such material can survive passage through interplanetary space, as well as passage through the Earth's atmosphere and the rigors of the associated deceleration and ablation thereby induced. That crater-produced ejecta can escape into space from the surface of the Moon and the surfaces of Mars and Mercury is aided by the fact that these worlds have relatively small escape velocity barriers and no substantial atmospheres. This latter condition may not have been true at all epochs over the age of the solar system, however, and there is a large body of evidence to indicate that the atmosphere of Mars was much more substantial in the past [10], and that the Moon may have undergone intermittent periods of atmospheric blanketing [6, 11]. Mercury has probably never supported a substantive atmosphere as a consequence of its close location to the Sun and because of its lack of any protective magnetic field. The former condition resulting in the typical speed of thermal motion being greater than the planet's escape velocity, and the latter effect allowing for a strong interaction with the solar wind and rapid gas removal.

In contrast to the Moon, Mercury and present-day Mars, material ejected from the Earth's surface will have always been launched under relatively high surface gravity conditions and under a substantive atmosphere. The purpose of this paper is to investigate these latter conditions, and to model and follow the path of a reversemeteorite as it moves through Earth's atmosphere. Accordingly, a computer code previously written to investigate the inward passage of a meteorite-producing meteoroid by Beech and Comte [12] has been modified so as to consider the out-ward going ejecta, from a launch location on the Earth's surface, as it moves through the atmosphere and on into cislunar space.

Given the similarity in mass, size and surface gravity of Venus and Earth, it is reasonable to ask why no suspected Cythean meteorites have ever been found. Certainly, Venus has not escaped impact collisions, and Cythean craters are discernible, for example, in the survey maps produced by the Magellan spacecraft [13]. For Venus, however, the answer lies entirely within the atmospheric covering, which with a surface density of order $65.5 \mathrm{~kg} / \mathrm{m}^{3}$ will result in the rapid ablation of any crater-produced ejecta even if imparted with a velocity in excess of the planet's escape velocity of $10.4 \mathrm{~km} / \mathrm{s}$. This result is brought-out by considering the integrated atmospheric mass per unit area: $M_{\text {atmos }}=P_{\mathrm{S}} /(g \sin \theta)$, where $P_{\mathrm{S}}$ is the surface pressure, $g$ is the surface gravity and $\theta$ is the trajectory angle to the local horizon. Accordingly, applying the rule-of-thumb [1] that a meteoroid of mass $M_{\text {met }}$ can be considered as "stopped", that is reduced from its cosmic encounter velocity to subsonic speeds, once it encounters more than $\eta>10$ times its own mass in the form of an atmospheric gas column, so the escape condition becomes

$$
\left(\pi R^{2}\right) M_{\text {atmos }}<\eta M_{\text {met }}
$$

where $R$ is the meteoroid radius. Condition (1) accordingly sets a size limit for escape as

$$
R_{\text {min }}>\frac{3}{4 \eta} \frac{P_{S}}{\varrho_{m e t} g \sin \theta}
$$

where $\rho_{\text {met }}$ is the meteoroid density. Table 1 provides a set of representative sizes and masses for objects that, upon being launched during a cratering event, might retain a substantial fraction of their ejection velocity at the top of the atmosphere.

Table 1. Minimum size and mass for potential debris ejection into the inner solar system. Column 1 is the body under consideration, and columns 2, 3 and 4 indicate the appropriate surface gravity, pressure and escape velocity. The calculations for $R_{\min }$ and $M_{\min }$ assume $\eta=10, \rho_{\text {met }}=3000 \mathrm{~kg} / \mathrm{m}^{3}$ (corresponding to stony/basaltic material) and that $\theta=45^{\circ}$.

\begin{tabular}{llllll}
\hline Body & $\boldsymbol{g}\left(\mathbf{m} / \mathbf{s}^{2}\right)$ & $\boldsymbol{P}_{\mathbf{S}}(\mathbf{P a})$ & $\boldsymbol{V}_{\text {esc }}(\mathbf{k m} / \mathbf{s})$ & $\boldsymbol{R}_{\min }(\mathbf{m})$ & $\boldsymbol{M}_{\min }(\mathbf{k g})$ \\
\hline Mercury & 3.7 & $1 \times 10^{-9}$ & 4.2 & 0.0 & 0.0 \\
Venus & 8.8 & $9.3 \times 10^{6}$ & 10.4 & 37.354 & $6.6 \times 10^{8}$ \\
Earth & 9.8 & $1.0 \times 10^{5}$ & 11.2 & 0.361 & 591.2 \\
Moon & 1.7 & $3 \times 10^{-10}$ & 2.4 & 0.0 & 0.0 \\
Mars & 3.7 & 600 & 5.0 & 0.005 & 0.002 \\
\hline
\end{tabular}

Table 1 indicates that for debris to potentially escape from the surface of Venus a minimum size some 75 meters across is required, while for the Earth the minimum escape size 
limit is about 0.7 meters in diameter. This result partially explains the complete lack of Cythean meteorites on Earth, while allowing for the presence of Hermian, Lunar and Martian meteorites within terrestrial collections. Note, however, that the radii indicated in table 1, column 5 will increase as the ejection angel decreases - this being a direct consequence of the greater atmospheric path length associated with low angle trajectories. In the remainder of this article the conditions for the ejection of terrestrial material, into the inner solar system, are investigated with particular attention being applied to the atmospheric passage of the crater-spalled material.

\section{The Crater Ejection Process}

Figure 1 provides a schematic cross-section for the various displacement and ejecta domains associated with a crater-forming impact. Some material, as indicated in the figure, is vaporized, some is melted, some is displaced and some is directly ejected. Of specific interest here, however, is the material located in the spallation zone. While material from the ejection zone suffers significant heating and shock pressure alteration, the material expelled from the spallation zone is accelerated to high speed in a relatively gentle manner and with little shock alteration [1, 14, 15]. This latter condition is of great importance since if the shock pressure exceeds that of the material tensile strength then extensive fragmentation will occur. The importance of the spallation zone has been particularly highlighted with respect to the ejection of material from the surface of Mars, and the production of potential Martian meteorites [14]. While shock alteration and heating effects are certainly discernable in all of the Martian meteorite groups, those within the Nakhlite group are especially noteworthy for how little shock processing they have experienced [8]. Indeed, petrological analysis of the Nakhlite meteorite MIL03346 indicates shock processing of no more than 0.1 GPa [16] - a shock pressure much smaller than the typical tensile strength, which is of order $1 \mathrm{GPa}$, for most rock and meteorite types. In terms of the lithopanspermia conveyorbelt operating between bodies within the solar system, it is additionally noted here that there is a growing body of evidence to indicate the pre-launch, that is in situ, alteration and etching of veins and micro-porosity regions, by (presumed) Martian microbes, is preserved in the Nakhla group of meteorites $[17,18]$. As to whether past stowaway microbes, transported within Martian meteorites, have survived to reach the Earth's surface, or indeed whether they seeded life on Earth, is currently unknown, but the topic has, and continues to be, keenly debated [19, 20, 21]. As far as the terrestrial component to the meteorite conveyor belt is concerned it is presumed that the larger the bodies that can be placed into interplanetary space, so the greater the likelihood that any microbial cargo will survive the launch, in-space journey and landing phases. It is the characteristic size of ejecta boulders, produced during an impact event, that is considered next.

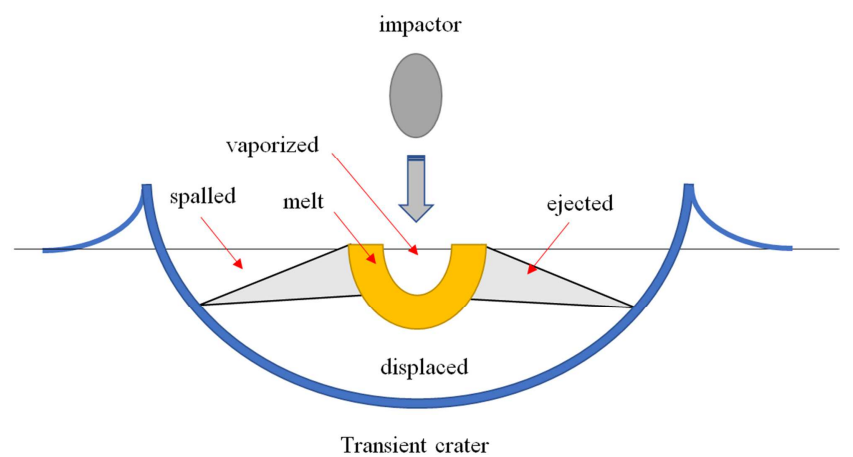

Figure 1. Schematic cross-section of a newly forming crater, indicating those zones that become vaporized, melted, ejected, shock altered and displaced, and spalled.

The location of the relatively less-aggressive, low-shock pressure spallation regions associated with transient crater excavation are illustrated in figure 1 . Melosh [1, 15] has studied the material ejection process in some considerable detail and has provided an analytic formula, based upon detailed hydrocode simulations, for the characteristic size of material boulders ejected from the spallation zone. Accordingly, the typical ejecta-size, $D_{B}$ is

$$
D_{B}=\frac{2 T a}{\rho_{\text {target }} V_{\text {eject }}^{2 / 3} V_{\text {imp }}^{4 / 3}}
$$

Where $T$ is the tensile strength of the target (surface) material, $\rho_{\text {target }}$ is the density of the target substrate, $V_{\text {imp }}$ is the impact velocity of the asteroid / comet, and $a$ is the radius of the impactor. The characteristic final-crater diameter, $D_{\mathrm{f}}$ produced on Earth by an impactor of radius $a$, density $\rho_{\text {imp }}$ and impact velocity $V_{\text {imp }}$, is given by the scaling relation described in Collins, Melosh and Marcus [22]

$$
D_{f}=0.782\left(\frac{\rho_{\text {imp }}}{\rho_{\text {target }}}\right)^{1 / 3} a^{0.78} V_{i m p}^{0.44}
$$

The final crater diameter on a body other than the Earth can be evaluated as $D_{f}\left(9.81 / g^{\prime}\right)^{-0.22}$ where $g$ ' is the surface gravity of the body (planet or moon) in question. There is an additional $\sin ^{1 / 3} \theta$ term, where $\theta$ is the impact angle relative to the horizon, that can be included, but for the sake of the discussion here this angle is taken as being $45^{\circ}-$ the most probable angle of entry or impact. Eliminating the impactor radius term $a$ between equations (3) and (4), however, provides for a relationship between crater size, ejecta velocity, characteristic ejecta size and impactor speed such that,

$$
D_{f}(k m)=0.022 D_{B}^{0.78} V_{e j e c t}^{0.52} V_{\text {imp }}^{1.48}
$$

where $D_{\mathrm{B}}$ is expressed in metres, $V_{\text {eject }}$ and $V_{\text {imp }}$ are expressed in units of $\mathrm{km} / \mathrm{s}$, and where it is assumed that $\rho_{\text {imp }}=\rho_{\text {target }}=$ $3000 \mathrm{~kg} / \mathrm{m}^{3}$, and that $T=1 \mathrm{GPa}$. Given a characteristic impactor speed of $V_{\text {imp }}=25 \mathrm{~km} / \mathrm{s}$, and $V_{\text {eject }}>V_{\text {escape }}$ (Earth's escape velocity) $=11.2 \mathrm{~km} / \mathrm{s}$, a 5 -metre diameter bolder could be launched and ejected into cislunar space from an impacting event capable of producing a crater about $30 \mathrm{~km}$ 
across. Alternatively, a $10 \mathrm{~km}$ diameter crater-producing event might potentially launch fragments with sizes of order $1-\mathrm{m}$ across into near-Earth space (see figure 2).

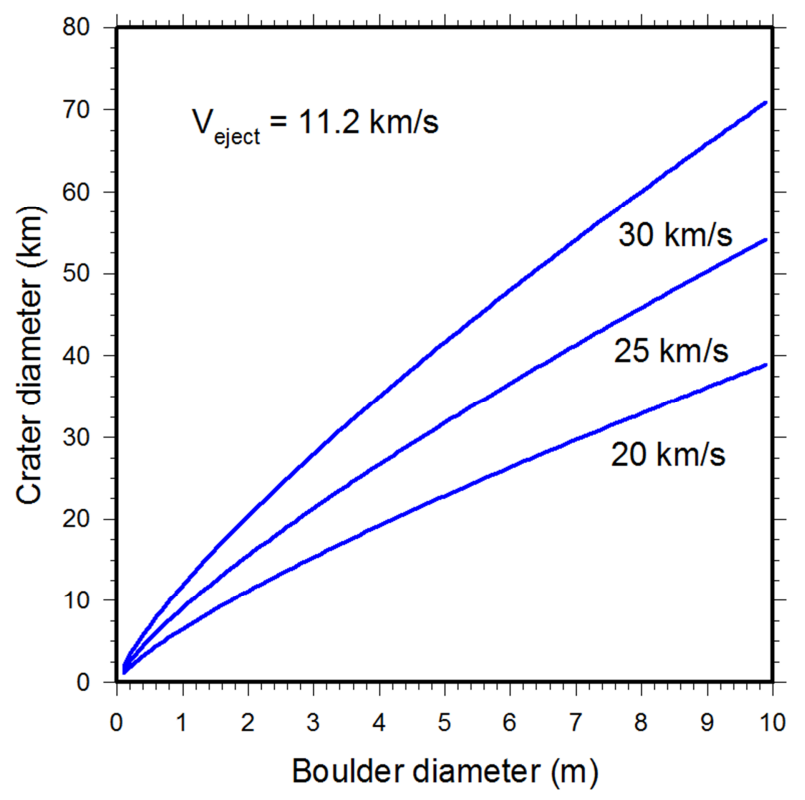

Figure 2. Crater size versus characteristic ejected boulder size for $20 \leq V_{\text {imp }}$ $(\mathrm{km} / \mathrm{s}) \leq 30$. In each case $V_{\text {eject }}$ is taken to be the Earth's escape velocity of $11.2 \mathrm{~km} / \mathrm{s}$.

The ejection velocity and size of the spalled material that has been described so-far has not taken into account passage through the Earth's atmosphere. At ejection speeds of order Earth's escape velocity or greater some heating and ablation is to be expected before the spalled ejecta potentially enters cis-lunar and/or interplanetary space. The ablation process will both reduce the size of the ejecta and slow its velocity, and the question becomes, what is the filtering-effect of the Earth's atmosphere upon material that might eventually impact upon the Moon.

\section{The Reverse-Meteorite Model}

The equations describing meteoroid ablation and deceleration have recently been described in detail by Beech and Comte [12]. In this study the equations are modified so as to follow the motion of ejecta launched from the Earth's surface. Accordingly, the equations describing the deceleration due to interactions with the Earth's atmosphere, the mass loss due to ablation, along with the down-range angle to the horizon $\theta$, ground distance $X$ and atmospheric height $h$ are:

$$
\begin{gathered}
\frac{d V}{d t}=-\Gamma \rho_{\text {atmos }}\left(\frac{A}{m}\right) V^{2}-g \sin (\theta) \\
\frac{d m}{d t}=-\left(\frac{\Lambda}{2 \zeta}\right) \rho_{a t m o s} A V^{3} \\
\frac{d \theta}{d t}=\left(\frac{1}{m V}\right)(-m g \cos (\theta))+\frac{V \cos (\theta)}{R+h} \\
\frac{d X}{d t}=\frac{V \cos (\theta)}{1+h / R}
\end{gathered}
$$

$$
\frac{d h}{d t}=+V \sin (\theta)
$$

Where $V$ is the velocity and $m$ the mass of the projectile; $\rho_{\text {atmos }}$ is the atmospheric density at height $h ; A$ is the crosssection area of the projectile (assumed spherical), $g$ is the acceleration due to gravity at height $h, R$ is the Earth's radius, and $\Lambda, \zeta$ and $\Gamma$ are the heat transfer coefficient, the ablation coefficient and the drag coefficient, respectively - the evaluation of these latter terms is described in Beech and Comte [12].

Critical to the study of reverse-meteorite dynamics and ablation is the state of the Earth's atmosphere following the impactor striking the ground. It is generally taken that the post-impact atmosphere will be highly perturbed from its normal state $[23,24,25]$, with the duration and amount of perturbation being impactor-size and energy dependent. Indeed, it is estimated that the high-speed vapor plume expanding away from the crater location carries away from between 25 to $50 \%$ of the total impact energy [25]. Two cases of plume behavior can be considered; the first case being where the downward motion of a large impactor plus the upward moving plume literally punch a short-lived (duration of order minutes) cavity through the atmosphere, effectively giving any solid ejecta an almost atmosphere-free ride into cis-lunar space. In this situation, the spalled ejecta (the reverse meteorite matter) will follow a purely ballistic trajectory into space and will suffer little to no ablation mass loss. In contrast, for small impactors where the expansion of the vapor plume is halted in the lower atmosphere (say at altitudes smaller than one atmospheric scale height, $H=8$ $\mathrm{km}$ ), the solid ejecta will encounter a largely unperturbed atmosphere. In this latter situation, some mass loss and deceleration of solid ejecta is inevitable and some degree of atmospheric filtering of smaller ejecta will occur. Melosh [24] has estimated the conditions under which the vapor plume is likely to tunnel through the atmosphere, and finds a specific constraint upon the impactor size such that for noplume entrapment

$$
D_{\text {blow-out }}>\frac{1}{2}\left(\frac{\rho_{\text {atmos }}}{\rho_{\text {imp }}}\right)^{1 / 3} H
$$

where $\rho_{\text {atmos }}$ is the atmospheric density close to the Earth's surface (say at height, $h=H / 4$ ). Taking $\rho_{\text {atmos }}=1.0 \mathrm{~kg} / \mathrm{m}^{3}$ at $h=2 \mathrm{~km}$, so equation (11) indicates that the vapor plume will burst through the Earth's atmosphere for impactors larger than $D_{\text {blow-out }} \approx 300 \mathrm{~m}$. Such an impactor will produce a crater of order 5-6 km in diameter (given a typical impactor speed of $V_{\mathrm{imp}}=25 \mathrm{~km} / \mathrm{s}$ [22]). Accordingly, the approach adopted in the following reverse-meteorite analysis is to consider two extreme scenarios. Scenario 1 assumes that there is no atmospheric alteration, with the reverse meteorite simulations requiring passage through a normal, non-perturbed, Earth atmosphere. Scenario 2 will assume a highly reduced atmospheric density profile with $\rho_{\text {atoms }}(h$, perturbed $)=\varepsilon$ $\rho_{\text {atoms }}(h$, non-perturbed), where $\varepsilon<1.0$ for all $h$.

Table 2 shows the results of applying the reverse meteorite 
model with a non-perturbed atmosphere, while table 3 shows the results for an atmosphere in which it is envisioned that the atmospheric density is negligible for the first $8 \mathrm{~km}$ (approximately one unperturbed atmospheric scale height) of flight with $\varepsilon=0.5$ thereafter. A range of initial sizes and ejection velocities have been considered, and a fragment is deemed to have escaped from the Earth's gravitational influence if it has a velocity greater than the Earth's escape velocity $\left(V_{\text {esc }}=11.2 \mathrm{~km} / \mathrm{s}\right)$ at $200 \mathrm{~km}$ altitude. The simulations leading to tables 2 and 3 assume a ground and fragment density of $2500 \mathrm{~kg} / \mathrm{m}^{3}$. For initial radii just a few meters across it is found that material can escape into cislunar space provided that the initial ejection angle is close to vertical (column 4 in each table), but, as would be expected, larger ejecta can escape along longer atmospheric paths, over a wider range of initial ejection angles, than is the case for smaller ejecta.

Table 2. Reverse meteorite model calculations for a non-perturbed atmosphere. Column 1 is the initial ejecta radius; column 2 is the final radius at $200 \mathrm{~km}$ altitude, and column 3 is the ground ejection velocity. Column 4 indicates minimum initial angle of ejection (angle to the horizon) that allows for atmospheric escape.

\begin{tabular}{llll}
\hline Initial radius & Final radius & $\mathbf{V}_{\text {eject }}(\mathbf{k m} / \mathbf{s})$ & Angle $(\mathbf{d e g})$. \\
\hline 1 & 0.96 & 42 & 88 \\
1 & 0.96 & 43 & 80 \\
1 & 0.96 & 44 & 76 \\
2 & 1.98 & 22 & 74 \\
2 & 1.98 & 23 & 65 \\
2 & 1.98 & 24 & 59 \\
3 & 2.99 & 18 & 67 \\
3 & 2.99 & 19 & 56 \\
3 & 2.98 & 20 & 49 \\
4 & 3.99 & 16 & 68 \\
4 & 3.99 & 17 & 52 \\
4 & 3.98 & 18 & 44 \\
5 & 4.99 & 15 & 66 \\
5 & 4.99 & 16 & 48 \\
5 & 4.98 & 17 & 39 \\
10 & 9.99 & 13 & 71 \\
10 & 9.99 & 14 & 38 \\
10 & 9.99 & 15 & 27 \\
10 & 9.98 & 16 & 22 \\
10 & 9.98 & 17 & 19 \\
\hline
\end{tabular}

Table 3. Same as table 2, but for a strongly perturbed atmosphere providing a negligible density for the first $8 \mathrm{~km}$ of flight and having a density reduction factor of $\varepsilon=0.5$ thereafter.

\begin{tabular}{llll}
\hline Initial radius & Final radius & $\mathbf{V}_{\text {eject }}(\mathbf{k m} / \mathbf{s})$ & Angle $(\mathbf{d e g})$. \\
\hline 1 & 0.99 & 16 & 67 \\
1 & 0.99 & 17 & 59 \\
1 & 0.99 & 18 & 53 \\
2 & 1.99 & 14 & 57 \\
2 & 1.99 & 15 & 47 \\
2 & 1.99 & 16 & 41 \\
3 & 2.99 & 13 & 58 \\
3 & 2.99 & 14 & 43 \\
3 & 2.99 & 15 & 36 \\
4 & 3.99 & 13 & 48 \\
4 & 3.99 & 14 & 36 \\
4 & 3.99 & 15 & 29 \\
5 & 4.99 & 13 & 41 \\
5 & 4.99 & 14 & 31 \\
\hline
\end{tabular}

\begin{tabular}{llll}
\hline Initial radius & Final radius & $\mathbf{V}_{\text {eject }}(\mathbf{k m} / \mathbf{s})$ & Angle $(\mathbf{d e g})$. \\
\hline 5 & 4.99 & 15 & 25 \\
10 & 9.99 & 12 & 47 \\
10 & 9.99 & 13 & 25 \\
10 & 9.99 & 14 & 18 \\
10 & 9.98 & 15 & 14 \\
\hline
\end{tabular}

Tables 2 and 3 reveal that irrespective of atmospheric conditions (non-perturbed, or strongly perturbed) it is harder to drive smaller, meter-sized fragments into cis-lunar space than larger multi-meter-sized fragments (this is effectively the situation described earlier in table 1). In general, the simulations indicate that smaller fragments require a larger initial velocity in order push through the atmosphere, losing most of their mass through ablation in the process, and they are additionally constrained to exit via a narrow range of (close to vertical) ejection angles for escape to take place. The simulations further suggest that it is the ejection of relatively large, multi-meter-sized, 5 to 20 -meter diameter, fragments that will most likely be placed into cis-lunar space as a consequence of terrestrial impact events. These results essentially follow expectation according to the observation that the larger ejecta fragments will have higher values of the ballistic coefficient, $B=m /(\Gamma A)$, than smaller ones. The higher the ballistic coefficient, so the easier it is for an object to pass through a given column of air (that is the smaller the resultant deceleration), and assuming that all ejecta is spherical in shape and composed of the same material, then the ballistic coefficient will scale directly as the ejecta radius.

The numerical simulations also indicate that for a fixed initial radius, the greater the ejection velocity, so the wider is the range in ejection angles allowing for escape to take place - that is the ejecta cone angle becomes larger. This latter effect will tend to favour material ejection into cis-lunar space from long-period comet impacts since the typical long period comet encounter speed will be of order $50-60 \mathrm{~km} / \mathrm{s}$, in contrast to the slower $20-30 \mathrm{~km} / \mathrm{s}$ associated with asteroids and short-period comet impacts [2]. Additionally, the simulations reveal that an increase in the density attributed to the terrestrial fragments, for a fixed size, results in a decrease in the ejection velocity required for the achievement of atmospheric escape (see table 4). This latter constraint favours the ejection of igneous (or crystalline) over sedimentary and loosely consolidated rock fragments into cis-lunar space.

Table 4. The effect of varying the fragment density (column 1) upon the minimum ejection velocity (column 2) required for escape into cis-lunar space. Column 3 shows the minimum ejection angle to the horizon. The fragment radius is fixed, in this example, to 2 meters and no atmospheric density perturbation is assumed (that is $\varepsilon=1$ ).

\begin{tabular}{lll}
\hline Density $\left(\mathbf{k g} / \mathbf{m}^{\mathbf{3}}\right)$ & Velocity $(\mathbf{k m} / \mathbf{s})$ & Angle (deg.) \\
\hline 1500 & 34 & 79 \\
2000 & 26 & 75 \\
2500 & 22 & 74 \\
3000 & 20 & 70 \\
3500 & 18 & 80 \\
4000 & 17 & 81 \\
\hline
\end{tabular}




\section{Lunar Landing}

Having once left the Earth's atmosphere behind, the reverse-meteorite enters into cis-lunar space, and indeed in terms of inner solar system impact targets, it is the Moon that first presents itself as a potential repository for terrestrial ejecta. While the Moon has no substantive atmosphere at the present epoch, it is not clear that this has always been the case. Indeed, it is highly probable that the Moon had at least a substantive primordial atmosphere as a consequence of post-formation outgassing (from its initial global magma ocean) and from an extended period of volcanic activity [5, 6, 11]. It is also entirely possible that the Moon has - supported short-lived atmospheres in the wake and disturbance of massive past impacts. It is also plausible that the Moon might be engineered in the (distant) future to support an artificially maintained atmosphere $[11,26]$. In this analysis, the assumption will be that the vast majority of terrestrial ejecta has in the past, and will continue to do so in the near-term future, encounter an atmosphere-less Moon. Such discussion is relevant, of course, in that the terrestrial ejecta encounter conditions will vary according to the existence of a lunar atmosphere or not. Specifically, the presence of an atmosphere, substantial or otherwise, will result in the deceleration of any in-coming terrestrial ejecta, thereby enhancing its likelihood of survival against vaporization and/or catastrophic fragmentation upon impact. The Moon's escape velocity is just $2.4 \mathrm{~km} / \mathrm{s}$ (recall table 1 ), and this sets a minimum impact speed for any terrestrial ejecta, the question to be addressed below, however, is over what encounter speed range might terrestrial ejecta survive intact upon lunar impact.

The shock pressures within the target (lunar regolith) and the projectile (terrestrial ejecta) materials following an impact can be investigated through an application of the planar impact approximation $[1,5]$. This technique enables a straightforward (analytic) determination of the maximum shock pressures that will be experienced by the impactor and the target during an impact event. Following Melosh [1], the velocity of the un-shocked projectile is taken as $\mathrm{u}_{\mathrm{i}} \equiv V_{\mathrm{imp}}$, and the relationship between the shock velocities $U_{t}$ and $U_{p}$ in the target and projectile are

$$
\begin{gathered}
\mathrm{U}_{\mathrm{t}}=\mathrm{C}_{\mathrm{t}}+\mathrm{S}_{\mathrm{t}} \mathrm{u}_{\mathrm{t}} \\
\mathrm{U}_{\mathrm{p}}=\mathrm{C}_{\mathrm{p}}+\mathrm{S}_{\mathrm{p}} \mathrm{u}_{\mathrm{p}}
\end{gathered}
$$

where the subscript labels $t$ and $\mathrm{p}$ correspond to target and projectile, respectively, and where $\mathrm{C}_{t}, \mathrm{~S}_{\mathrm{t}}, \mathrm{C}_{\mathrm{p}}$ and $\mathrm{S}_{\mathrm{p}}$ are empirically derived constants set according to the target and projectile materials (see table 5 below). The velocities $u_{t}$ and $\mathrm{u}_{\mathrm{p}}$ are determined as $\mathrm{u}_{\mathrm{t}}=\mathrm{u}_{\mathrm{i}}-\mathrm{u}_{\mathrm{p}}$, where $\mathrm{u}_{\mathrm{p}}$ is the velocity behind the shock front, and $u_{t}$ is the change in the velocity across the shock front propagating into the target substrate. Once $u_{t}$ is known then the shock velocities in both target and projectile can be determined, and thereafter the shock pressures $\mathrm{P}_{\mathrm{t}}=\rho_{\mathrm{t}} \mathrm{U}_{\mathrm{t}} \mathrm{u}_{\mathrm{t}}$ and $\mathrm{P}_{\mathrm{p}}=\rho_{\mathrm{p}} \mathrm{U}_{\mathrm{p}} \mathrm{u}_{\mathrm{p}}$ can be determined, where $\rho_{\mathrm{t}}$ and $\rho_{\mathrm{p}}$ are the densities of the target and projectile materials, respectively. The Hugoniot equations that describe the conservation of mass, energy and momentum across the shock front are used to solve for $u_{t}$ in the planar approximation, with,

$$
u_{t}=\frac{-B+\sqrt{B^{2}-4 A C}}{2 A}
$$

where

$$
\begin{aligned}
& \mathrm{A}=\rho_{\mathrm{t}} \mathrm{S}_{\mathrm{t}}-\rho_{\mathrm{p}} \mathrm{S}_{\mathrm{p}} \\
& \mathrm{B}=\rho_{\mathrm{t}} \mathrm{C}_{\mathrm{t}}+\rho_{\mathrm{p}} \mathrm{C}_{\mathrm{p}}+2 \rho_{\mathrm{p}} \mathrm{S}_{\mathrm{p}} \mathrm{u}_{\mathrm{i}} \\
& \mathrm{C}=-\rho_{\mathrm{p}} \mathrm{u}_{\mathrm{i}}\left(\mathrm{C}_{\mathrm{p}}+\mathrm{S}_{\mathrm{p}} \mathrm{u}_{\mathrm{i}}\right)
\end{aligned}
$$

With the constants $\mathrm{C}_{\mathrm{t}}, \mathrm{S}_{\mathrm{t}}, \mathrm{C}_{\mathrm{p}}$ and $\mathrm{S}_{\mathrm{p}}$ described, so $\mathrm{u}_{\mathrm{t}}$ and the shock pressure experienced by the target and the projectile can be calculated for any adopted value of the impact velocity, $u_{i}$. Table 5 provides a set of representative equation of state parameters for projectile and lunar target material. The lunar target material is taken to be that of a regolith. In this case rather than the impactor striking bedrock, it is envisioned as impacting a layer of unconsolidated rock fragments. Indeed, samples of lunar regolith have been directly studied in the laboratory as a result of the Apollo Moon Landings [7]. Such studies indicate that the lunar regolith is a fine-grained, cohesive mixture of igneous rock fragments, crystalline impact melts, breccias and micrometeorite impact glasses (agglutinates) with a typical porosity of $50 \%$. There is no direct equivalent substrate to the lunar regolith on Earth, but dry, fine-sand has similar physical properties [27]. Both high- and low-pressure equation of state parameters are considered for the regolith material, with the high-pressure parameters allowing for the situation in which dynamic compaction, bulk modulus and density changes occur during the impact process.

Table 5. Equation of state parameters for specific projectile materials and lunar regolith. Column 1 and 2 identify the composition and density of the target and projectile materials. Columns 3 and 4 provide the equation of state constants - Data from ${ }^{1}$ Melosh [1], ${ }^{2}$ Syono et al. [28], ${ }^{3}$ Crawford et al. [5] and ${ }^{4}$ Brown et al. [29].

\begin{tabular}{llll}
\hline Material & $\boldsymbol{\rho}\left(\mathbf{k g} / \mathbf{m}^{\mathbf{3}}\right)$ & $\mathbf{C}$ & S \\
\hline Granite $^{1}$ & 2630 & 3680 & 1.24 \\
Basalt $^{1}$ & 2860 & 2600 & 1.62 \\
Forsterite $^{2}$ & 3275 & 6260 & 1.12 \\
Regolith (low P) $^{3,4}$ & 1660 & 1700 & 0.46 \\
Regolith (high P) $^{3,4}$ & 1660 & 2100 & 1.1 \\
\hline
\end{tabular}

Figure 3 shows the results from a set of calculations in which various projectile materials composed of either granite (black line), basalt (red line) or forsterite (blue line, this being representative of an ultramafic cumulate rock) are envisioned to impact the lunar regolith at a range of velocities $V_{\text {imp }}=\mathrm{u}_{\mathrm{i}}>2.4 \mathrm{~km} / \mathrm{s}$. For each projectile composition the upper set of curves correspond to the highpressure equation of state parameters, while the lower set of curves correspond to the low-pressure domain (see table 5). 


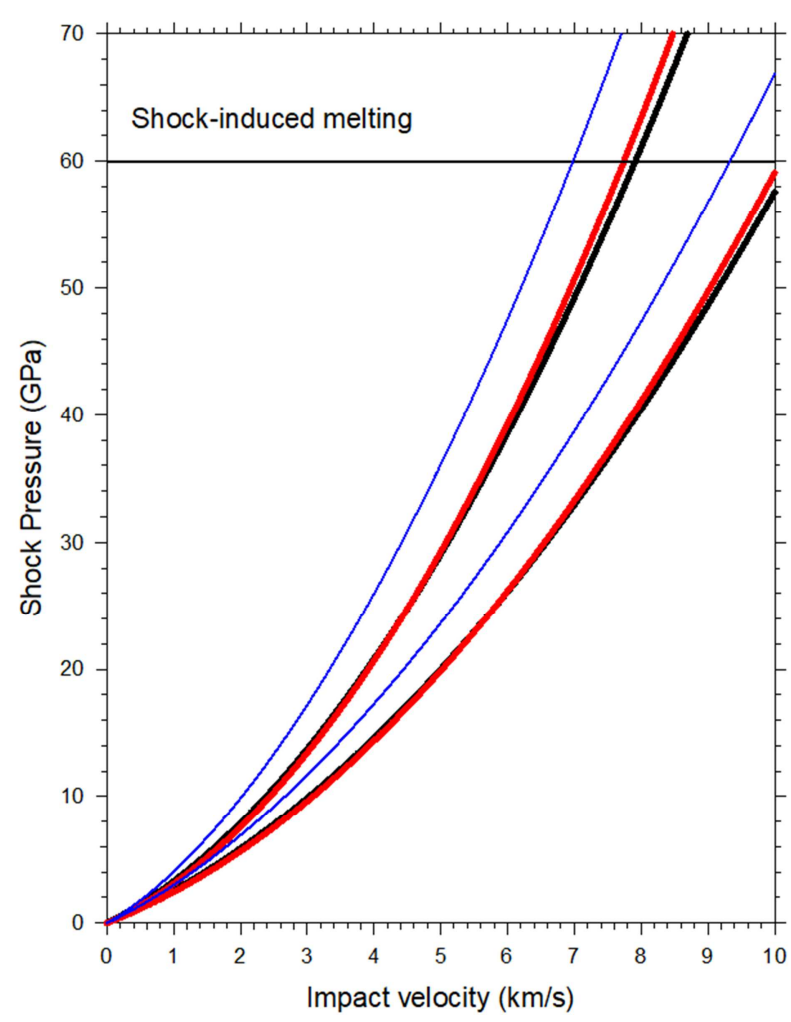

Figure 3. Shock pressure in the projectile for lunar regolith impacts.

The upper and lower curves for each projectile type are determined according to the high- and low-pressure regolith equation of state parameters (as given in table 5). The black lines correspond to granite projectiles, red basaltic projectiles and blue forsterite projectiles. The horizontal line at $60 \mathrm{GPa}$ indicates the general limit above which total projectile melting is expected to take place.

Figure 3 indicates that material ejected from the Earth's surface, can survive without shock melting impact upon the Moon's surface over a wide range of impact velocities - from at least $2.5 \mathrm{~km} / \mathrm{s}$ to of order $7 \mathrm{~km} / \mathrm{s}$. Such accumulated material can rightly be described as being captured terrestrial meteorites, and accordingly such objects may be searched for during future lunar surveys, and within those lunar breccias returned to Earth during the Apollo missions. Given a characteristic boulder size of 5-m across, and an impact speed of $2.5 \mathrm{~km} / \mathrm{s}$, so equation (4), modified according to the Moon's surface gravity $g^{\prime}=1.62 \mathrm{~m} / \mathrm{s}^{2}$, reveals that terrestrial meteorites might produce lunar impact craters with diameters up to of order 2 kilometers across.

Since it is envisioned that any terrestrial ejecta accumulated by the Moon will have undergone some surface ablation processing while exciting the Earth's atmosphere, one of the key features that will make terrestrial meteorite fragments standout against the lunar regolith substrate will be the presence of a fusion crust. Fusion crust glass, however, is fragile and will, if the meteorite is not fully buried, begin to suffer the weathering and degradation effects of micrometeoroid impact erosion. The characteristic micrometeoroid erosion rate at the Moon's surface is estimated to be of order $1 \mathrm{~mm}$ per million years [30], and, accordingly, any fusion crust, if permanently exposed at the surface, is not likely to last much longer than a few millions of years from the time of accumulation. That terrestrial material can survive for very long intervals of time within the lunar regolith, and remain identifiable, is indicated by the Apollo 14 Moon rock sample \#14321 studied by Bellucci et al. [7], where it is estimated that the terrestrial clast is at least 3.9 billion years old.

The arrival rate of terrestrial meteorites upon the Moon is likely to be stochastic in the sense that the ejection mechanism itself is only quasi-periodic and depends upon the production of a 10 to $30-\mathrm{km}$ or larger crater on the Earth. Such impacts occur at a global rate of about 1 per 10-millionyears. One of the most recent, large, terrestrial impact events that may well have produced a significant flux of terrestrial meteorites is that of the Chicxulub impact which occurred some 65 million years ago [2]. Terrestrial meteorites accumulated on the Moon from this particular event will have now lost some 6 to $7 \mathrm{~cm}$ of their original outer layers, if permanently exposed at the Moon's surface, due to micrometeoroid impacts. The youngest known terrestrial craters with diameters in the $10 \mathrm{~km}$ diameter range are those of Zhamanshin in Kazakhstan and Bosumtwi in Ghana these craters having estimated ages of $0.9 \pm 0.1$ and $1.07 \pm$ 0.11 million years, respectively [2,3]. Terrestrial meteorites on the Moon from these impacts may well still show a fusion crust.

While the presence of a fusion crust would be highly diagnostic of a terrestrial meteorite on the Moon, other distinctive features might also be recognized in older accumulates. The Moon, for example, is entirely devoid of water at the surface, and is depleted in volatile elements, where as terrestrial material is not [5, 6]. Accordingly, infrared spectroscopy could be used to look for hydrated silicates - any such detection being a clear signature of nonlunar origin. It is also the case that terrestrial meteorites could display evidence of biomarkers - that is microfossils and/or complex organic structures and imprints. These latter signatures being evidenced by microscopic studies in situ to the Moon's surface by an automated rover, or in the laboratory (either on Earth or a future Moon base). The terrestrial origin of material in the \#14321 Apollo 14 sample [7], for example, was betrayed through the analysis of its zircon crystal inclusions, and by evaluating the oxygen fugacity of the material's formation environment. Such detailed chemical analysis may eventually be available in a Moon-base laboratory, although that day is still far-off in our future.

\section{Discussion}

The search for terrestrial meteorites upon the Moon's surface offers an incredible opportunity to test several aspects of the lithopanspermia hypothesis. Not least the confirmation that potentially life-bearing material can be ejected from the surface of the Earth and thereafter be transported to a new, not necessarily nurturing, location within the solar system. It 
has long been suggested that life may have been seeded on Earth through the exchange of material from a young Mars, but it is here argued that the reverse process must also apply. With the near-term prospects of both in situ lunar surface surveys, as well as future astrobiology missions to Mars (beginning with the up-coming ExoMars rover mission due for launch in 2020), a new and potentially defining era of solar system and origins of life exploration is primed to begin. On the Moon, at least in principle, terrestrial meteorites might still exist and contain within their matrix information pertaining to the very first microbes that evolved on the Earth - their parent terrestrial material possibly being launched during the late heavy bombardment [7, 31], and at epochs comparable in ages to the ancient microbes found, for example, within the Apex cherts located within the Australian Pilbara Craton (dated to some 3.5 billion years old in age [32]), and the Nuvvuagittuq Belt in Quebec, Canada (dated to 3.8 billion years old in age [33]). On Mars the possible near-future detection of either fossil or still active microbes [34] will allow for potential cross-referencing with terrestrial microbes [35, 36], either supporting thereby the lithopanspermia hypothesis (and demonstrating the great resilience of microbial life against extinction [37]), or indicating secondary biogenesis. It is additionally not inconceivable that distant-future exploration of the surface of Venus might find evidence for early microbial life (corresponding to the time interval prior to the moist greenhouse loss of its ocean in the first half-billion years following its formation) - allowing for further crossreferencing with terrestrial, and possible Mars, microbes being transported to Venus via the lithopanspermia conveyor-belt [38, 39].

Clearly, not all of the material ejected into cis-lunar space must be accreted by the Moon, and various sets of detailed calculations indicate that a good fraction of ejected material will eventually fall back to Earth $[40,41]$. This opens-up the possibility of terrestrial meteorites being located upon the Earth. Recognizing such objects will be difficult since they will not have a distinct lithology and/or chemical composition to that of ordinary terrestrial rock [42]. Terrestrial meteorites will be distinct from tektites, however, in the sense that they will not have undergone complete melting. Moreover, terrestrial meteorites will, at least in the case of non-sedimentary material, support a fusion crust - a consequence of their having passed through the Earth's atmosphere twice. Given the rapid erosion and weathering time that applies to any fusion crust, however, it is not overly surprising that no terrestrial meteorites have been recognized to this date. Since terrestrial meteorites will only follow in the wake of a large, $10-30 \mathrm{~km}$ diameter, crater forming event, they are not likely to be commonly found on Earth's surface, and presumably the best chance of finding such objects is to survey those specific geological strata that existed at the time of a known impact. Under these circumstances it might be hoped that re-accreted terrestrial meteorites could have been preserved as isolated fossil-like impactites [43, 44, 45].

\section{Conclusions}

It has been shown that material, with sizes in excess of several meters across, ejected from the surface of the Earth, as a result of an impact cratering event, can survive atmospheric passage and be launched into cis-lunar space. It is also shown that this same material can potentially survive impact into the lunar regolith without undergoing catastrophic shock vaporization - accordingly allowing for the implantation of bone fide terrestrial meteorites upon the Moon's surface. One of the key identification features of such terrestrial meteorites, resulting from their flight through Earth's atmosphere, will be the retention of at least some fusion crust. Given a gentle enough landing ( $V_{\mathrm{imp}}<7 \mathrm{~km} / \mathrm{s}$ ), the fusion crust of a terrestrial meteorite, even if permanently exposed, might remain identifiable on a timescale of order several million years, given the present-day estimates for the micrometeorite abrasion rate at the Moon's surface. In a broader context it has also been argued that terrestrial meteorites in the past, present and future, must be considered as important lithopanspermia vectors, not only for the distribution of microbial life throughout the solar system but also into interstellar space as well.

\section{References}

[1] H. J. Melosh 1989. Impact Cratering - a geological process. Oxford University Press, Oxford.

[2] M. Beech, I. M. Coulson, and M. Comte 2018. Lithopanspermia - the terrestrial input during the past 550 million years. American Journal of Astronomy and Astrophysics, 6(3), 81-90.

[3] Earth Impact Database: http://www.passc.net/EarthImpactDatabase/.

[4] B. M. Simonson, and P. G. Glass 2004. Spherule layersrecords of ancient impacts throughout Earth's history. Annual Review of Earth and Planetary Science, 32, 329-361.

[5] I. A. Crawford, et al. 2008. On the survivability and detectability of Terrestrial Meteorites on the Moon. Astrobiology, 8, 242-252.

[6] D. Schultze-Makuch and I. A. Crawford 2018. Was there an early habitable window for Earth's Moon? Astrobiology, 18, 1-4.

[7] J. J. Bellucci, et al. 2019. Terrestrial-like zircon in a clast from an Apollo 14 breccia. Earth and Planetary Science Letters, 510, 173-185.

[8] L. E. Nyquist et al. 2001. Ages and geological histories of Martian meteorites. In Chronology and Evolution of Mars, 96, 105-164.

[9] F. M. McCubbin, and T. J. McCoy 2016. Expected geochemical and mineralogical properties of meteorites from Mercury: inferences from MESSENGER data. The $79^{\text {th }}$ Annual meeting of the Meteoritical Society. 6242.pdf.

[10] H. Lammer, et al. 2018. Origin and evolution of the atmospheres of early Venus, Earth and Mars. Astronomy and Astrophysics Review, 26:2. 
[11] M. Beech 2008. Meteors over the Moon. WGN, the Journal of the IMO. 36:2, 33-36.

[12] M. Beech, and M. Comte 2018. The Chant meteor Procession of 1913 - towards a descriptive model. American Journal of Astronomy and Astrophysics, 6 (2), 31-38.

[13] G. G. Schaber et al. 1992. Geology and distribution of impact craters on Venus: what are they telling us? JGR Planets, 97 E8, 13257-13301.

[14] A. M. Vickery and H. J. Melosh 1987. The large crater origin of SNC meteorites. Science, 237, 738-743.

[15] H. J. Melosh. 1988 The rocky road to panspermia. Nature, $332,687-688$

[16] N. Artemieva and B. Ivanov 2004. Launch of Martian meteorites in oblique impacts. Icarus, 171, 84-101.

[17] D. S. McKay et al. 2006. Observation and analysis of in situ carbonaceous matter in Nakhla. Lunar and Planetary Science meeting XXXVII, 2251.pdf.

[18] L. M. White, E. K. Gibson, K. L. Thomas-Keprta, S. J. Clemett and D. S. McKay 2014. Putative indigenous carbonbearing alteration features in Martian meteorite Yamato 000593. Astrobiology, 14, 170-181.

[19] D. S. McKay et al. 1996. Search for past life on Mars: possible relic biogenic activity in Martian meteorite ALH84001. Science, 273, 924-930.

[20] R. A. Kerr 1998. Requiem for life on Mars? Support for microbes fades. Science, 282, 1398-1400.

[21] K. L. Thomas-Keprta, S. J. Clemett, D. S. McKay, E. K. Gibson, and S. J. Wentworth 2009. Origins of magnetite nanocrystals in Martian meteorite ALH84001. Geochimica et Cosmochimica Acta, 73, 6631-6677.

[22] G. S. Collins, H. J. Melosh, and R. A. Marcus 2005. Impact effects program. Meteoritics and Planetary Science, 40, 817840 .

[23] W. I. Newman, E. M. D. Symbalisty, T J. Ahens, and E. M. Jones 1999. Impact erosion of planetary atmospheres: some surprising results. Icarus, 138, 224-240.

[24] H. J. Melosh. 1990. Vapor plumes: a neglected aspect of impact cratering. Meteoritics, 25, 386.

[25] H. J. Melosh, and A. M. Vickery 1989. Impact erosion of the primordial atmosphere of Mars. Nature, 338, 487-489.

[26] R. R. Vondrak 1974. Creation of an artificial lunar atmosphere. Nature, 248, 657-659.

[27] E. N. Slyuta 2014. Physical and Mechanical Properties of the Lunar Soil (A review). Solar System Research. 48, 330-353.

[28] Y. Syono, T. Goto, J-I. Sato, and H. Takei 1981. Shock compression measurements of single - crystal forsterite in the pressure range 15-93 GPa. Journal of Geophysical Research solid Earth, 86, B7, 6181-6186.
[29] J. L. Brown, et al. 2007. Shock response of dry sand. Sandia Report: SAND2007-3524.

[30] H. Hiesinger and J. Head III 2006. New Views of lunar geoscience: an introduction and overview. In New Views of the Moon, B. Jolliff, M. Wieczorek, C. Shearer and C. Neal (Eds.). Mineralogical Society of America, Virginia. p. 28.

[31] A. Mann 2018. Cataclysm's End. Nature, 553, 393-395.

[32] J. W. Schopf et al., 2017. SIMS analyses of the oldest known assemblage of microfossils document their taxon-correlated carbon isotope compositions. Proceedings of the National Academy of Sciences, 201718063 DOI: 10.1073/pnas. 1718063115 .

[33] M. J. Dodd, et al. 2017. Evidence for early life in Earth's oldest hydrothermal vent precipitates. Nature, 543, 60-63.

[34] G. Galletta, G. Bertoloni and M. D'Alessandro 2010. Bacterial survival in Martian conditions. https://arxiv.org/abs/1002.4077.

[35] R. J. Worth, S. Sigurdsson, and C. H. House 2013. Seeding life on the Moons of the outer planets via lithopanspermia. Astrobiology, 13, $1155-1165$.

[36] A. K. Pavlov et al., 2016. Was Earth ever infected by Martian biota? Clues from radioresistant bacteria. Astrobiology, 6, 911-918.

[37] D. Sloan, R. A. Batista and A. Loeb 2017. The resilience of life to astrophysical events. Nature, scientific reports, 7:5149.

[38] H. Morowitz and C. Sagan 1967. Life in the clouds of Venus. Nature, 215, 1259-1260.

[39] C. S. Cockell 1999. Life on Venus. Planetary and Space Science, 47, 1487-1501.

[40] M. Reyes-Ruiz et al. 2012. Dynamics of escaping Earth ejecta and their collision probability with different solar system bodies. Icarus, 220, 777-786.

[41] B. Gladman, L. Dones, H. Levison, and J. Burns 2005. Impact seeding and reseeding in the inner solar system. Astrobiology, $5,483-496$.

[42] M. J. Simms 2011. Where are all the terrestrial meteorites. $74^{\text {th }}$ Annual Meteoritical Society meeting, 5474. pdf.

[43] B. Schmitz, M. Tassinari, and P. Peucker-Ehrenbrink 2001. A rain of ordinary chondrite meteorites in the early Ordovician. Earth and Planetary Science Letters, 194, 1- 15.

[44] P. R. Heck, et al. 2007. Cosmogenic and solar noble gasses in $470 \mathrm{Ma}$ fossil meteorites and micrometeorites from the Lchondrite parent-body break-up. $70^{\text {th }}$ Annual Meteoritical Society Meeting, 5027. pdf.

[45] F. T. Kyte, 1998. A meteorite from the Cretaceous/Tertiary boundary. Nature, 396, 237-239. 